\title{
SPACE EXPERIMENTS ON-BOARD OF LOMONOSOV MISSION TO STUDY GAMMA-RAY BURSTS AND UHECRS
}

\author{
A.M. Amelushkin ${ }^{1}$, V.V. Bogomolov ${ }^{1}$, V.V. Benghin ${ }^{1}$, G.K. Garipov ${ }^{1}$, \\ E.S. Gorbovskoy ${ }^{2}$, B. Grossan ${ }^{3}$, P.A. Klimov ${ }^{1}$, B.A. Khrenov ${ }^{1}$, J. Lee ${ }^{4}$, \\ V.M. Lipunov ${ }^{2}$, G. Na ${ }^{4}$, M.I. Panasyuk ${ }^{1}$, I.H. Park ${ }^{4}$, V.L. Petrov ${ }^{1}$, \\ G.F. Smoot ${ }^{3}$, S.I. Svertilov ${ }^{1}$, Yu. Shprits ${ }^{5}$, N.N. Vedenkin ${ }^{1}$ \\ and I.V. Yashin ${ }^{1}$
}

\begin{abstract}
The number of experiments on-board Lomonosov spacecraft are preparing now at SINP MSU in co-operation with other organisations. The main idea of Lomonosov mission is to study extreme astrophysical phenomena, such as cosmic gamma-ray bursts and ultrahigh energy cosmic rays. These phenomena connect with processes occurred in very distant astrophysical objects of the Early Universe and give us information about first stages of Universe evolution. Thus, the Lomonosov mission scientific equipment includes several instruments for gamma-ray burst observation in optics, ultra-violet, X-rays and gamma-rays and the wide aperture telescope for ultra-high energy particle study by detection of ionisation light along its tracks in the atmosphere. The main parameters and a brief description of these instruments are presented.
\end{abstract}

\section{Introduction}

Studies of extremely high energy and power processes such as ultra-high energy cosmic rays (UHECR) and cosmic gamma-ray bursts (GRB) are of great importance

\footnotetext{
${ }^{1}$ D.V. Skobeltsyn Institute of Nuclear Physics of M.V. Lomonosov Moscow State University, Leninskie Gory, 1/2, 119991 Moscow, Russia

${ }^{2}$ P.K. Shternberg Astronomical Institute of M.V. Lomonosov Moscow State University, Universitetskii prosp. 17, 119992 Moscow, Russia

${ }^{3}$ Berkeley Center for Cosmological Physics, Berkeley, California, USA

${ }^{4}$ Department of Physics, Sungkyunkwan University, Seobu-ro, Jangangu, Suwonsi, Gyeongido 440-746, Korea

${ }^{5}$ Inst. of Geophysics and Planetary Physics, UCLA, 405 Hilgard Ave / 7127, Los Angeles, CA, USA
} 
not only in order to understand these phenomena, but also to develop the theory of the Early Universe.

Gamma-ray bursts are observed as short (from dozens of milliseconds up to dozens of seconds) pulses of gamma-fluxes up to the energy of at least $10^{9} \mathrm{eV}$. Discovered in $60 \mathrm{~s}$ years of 20th century they are still at the cutting edge of astrophysics. These phenomena being the most powerful in the Universe occur not only in gamma-range, but also in optics and UV. The power of the explosion of these bright astrophysical objects achieves $10^{51}-10^{53} \mathrm{erg} / \mathrm{s}$. GRB optical emission lasts up to several hours or even days as an afterglow, which appears after a giant explosion in the external shock wave expanding in the interstellar medium and stellar wind of the exploded star. Probably, it is due to the collapse of a fast-rotating very massive star to a black hole in the case of so-called longduration (more than a few seconds) bursts or merging of neutron stars in tight binary system in the case of so-called short-duration (less than a second) bursts. However, these models are under discussion and the nature of these extraordinary phenomena is still unknown. Due unusually powerful brightness of GRBs, studying their properties allows the researchers to look in the epoch of the early Universe, i.e. to study evolution of the stars and the stellar populations within the wide range of redshifts from $\mathrm{z} \sim 0.1$ up to $\mathrm{z} \sim 15-20$, it is more than $98 \%$ of the age of our Universe.

The other extreme phenomena in the Universe are ultra-high energy cosmic rays, which are most likely produced by the Active galactic nuclei (AGN). The fundamental problem is to estimate maximal particle energy, to which they could be accelerated in such sources, and whether there is a maximum energy to which particles can be accelerated in the Universe. Because AGN are very distant objects, UHECR go a long way before coming to the Earth. During their propagation UHECR lose energy due to photo-production of secondary particles (mostly pions) on the microwave background photons. It leads to a natural limit of observable cosmic ray particle energy and to the UHECR energy spectrum cut-off at the photo-production energy threshold, i.e. about $5 \cdot 10^{19}-10^{20} \mathrm{eV}$ (GZK (Greisen-Zatsepin-Kuzmin) - cut-off). However, so far we have only limited and contradictory information about the energy spectrum and composition of the cosmic particles at extremely high energies. Thus, it is not possible to make any final conclusion about the nature of UHECR, their sources location and mechanism of acceleration.

\section{Scientific objectives}

The mentioned above problems of extreme phenomena studies dictate the scientific objectives of considerable space experiments and suggest a specific set of appropriate instruments.

The following problems should be studied during the Lomonosov mission:

- detection of GRBs within optical and gamma ranges especially in order to study the optical prompt emission and precursors; 
- studies of UHECR (5 $\left.10^{19}-10^{20} \mathrm{eV}\right)$ near the GZK energy spectrum cut-off;

- studies of the transient luminous phenomena in the upper atmosphere, started during the previous MSU space projects "Universitetsky - Tatiana" and "Universitetsky - Tatiana - 2" (Garipov et al. 2005; Garipov et al. 2010);

- detection of the magnetospheric particles, which are the possible sources of transient and quasi-stationary phenomena in the upper atmosphere within the X-ray and optical ranges.

The objective of GRB studies during the Lomonosov mission is to accomplish simultaneous burst detection within the gamma-rays and optics ranges along with possibility of obtaining prompt emission as well as precursor light curves. This possibility provides unique information about GRB central engine functioning. On this way we plan to use the successive experience of ground-based systems of wide field cameras and robotic telescopes MASTER, which had detected the prompt emission of several GRBs in September, 2010 (Gorbovskoy et al. 2011). The point is to use the co-aligned GRB gamma-ray monitor detectors and wide field optical cameras, which should be operated continuously and store the data on trigger from the GRB gamma-ray monitor. In this case the field of view (FOV) of the optical camera will be inside the FOV of gamma-ray detector and there will not need to redirect the optical system. Thus, the time delay between optic and gammaray signals will be zero, and even the event pre-history including the possible precursors could be recorded. Another approach is based on fast re-orientation technique using MEMS technology or very fast (during less than $1 \mathrm{~s}$ ) rotating mirror. In this case the source indication for the optical system is given by the trigger from X-ray imager.

Another goal of the Lomonosov mission is UHECR studies. The Earth's atmosphere will be used as a "detector" of UHECR, which produce cascades of secondary particles, i.e. extensive air showers (EAS), which can provide us information about the primary particle parameters. The bulk of secondary particles in EAS ionize molecules and atoms of atmospheric nitrogen and oxygen and lead to the so-called ionization glow, which is most intensive along the EAS axis and looks like a point source moving with light velocity during very short time (tens microseconds). The ionization light intensity provides information about the energy of primary particle, while direction of EAS track follows arrival direction of primary particle. Thus, an instrument capable to image the UHECR ionization track is necessary.

Such instrument will be also able to detect so-called "transient luminous events" (TLEs) in the upper atmosphere. The nature of TLEs is probably associated with atmospheric electricity phenomena. During the high-altitude electric discharges between the clouds and ionosphere (at altitudes of 10-70 km) short-time (with duration 1-100 milliseconds) bursts of electromagnetic radiation within wide spectral range (from visual light up to UV and even X-rays and gamma-rays) are observed. Current experimental data about discharges in the upper atmosphere have shown that these phenomena are global, number of discharges and the energy released in 
these discharges are so high that we can expect certain relations between discharge phenomena and other geophysical phenomena.

Studies of charge particle fluxes in the near-Earth space and especially the high energy magnetospheric electrons could be considered as an associated goal of the mission. Radiation environment at low altitudes (less than $\sim 500-600 \mathrm{~km}$ ) is basically determined by the fluxes of quasi-trapped and precipitated particles of the Earth's radiation belts and the solar particles penetrating mainly into the polar caps regions. The scientific payload of the "Lomonosov" satellite will include a complex of instruments for the studies of the processes of charged particles penetration into the upper atmosphere of Earth and for the analysis of the radiation conditions at low altitudes.

\section{Instrumentation}

Scientific equipment installed on-board the "Lomonosov" satellite includes a number of instruments intended to study the scientific cases mentioned above:

- set of instruments for GRB studies including gamma-ray monitor BDRG, optical wide-field cameras SHOCK and UFFO instrument consisting of UV and X-ray telescopes;

- optical wide aperture telescope TUS for imaging of the UHECR tracks in the atmosphere;

- set of instruments for studies of energetic particle fluxes in the near-Earth space including magnetometer, high energy electron detector ELFIN and charge and neutral particle monitor DEPRON.

\subsection{Space telescope TUS}

Orbital telescope TUS (Russian abbreviation for "Tracking Instrument") is intended for observations of UV (300-400 nm wavelength) bursts in the night atmosphere of Earth.

Detector consists of two main parts: mirror-concentrator with area of $1.8 \mathrm{~m}^{2}$ (Fig. 2) and photo detector composed of 256 pixels, located at the mirror focus (Figs. 1 and 3). TUS technological parameters are: mass $\sim 60 \mathrm{~kg}$, power consumption $\sim 65 \mathrm{~W}$, data rate 250 Mbytes/day.

Mirror- concentrator is designed as sum of the central parabolic mirror and 11 parabolic rings focusing a parallel beam to one focal point. In this design thickness of the mirror construction is small $(3 \mathrm{~cm})$ which is important for mirror implementation into satellite construction. Mirror focal distance is $1.5 \mathrm{~m}$. The mirror is cut to hexagonal segments with a diagonal of $63 \mathrm{~cm}$. Mirror segments are made of carbon plastic strengthened by a honey comb aluminum plate so that the mirror construction is thermally stable in a wide range of temperatures. Mirror surface is obtained as plastic replicas of aluminum press forms (one for central mirror part and one for 6 lateral parts). 
Plastic mirror surface is covered by an aluminum film and protected by a $\mathrm{SiO}_{2}$ coat based on vacuum evaporation. Reflectivity of the mirror surface at wavelength $350 \mathrm{~nm}$ (average for the atmosphere fluorescence) is $85 \%$. Expected life time of the mirror is not less than 3 years.

TUS mirror passed various space qualification and optical tests. These tests show stability of optical quality of the mirror in space conditions.

Photo detector pixels are photomultiplier tubes PMT R1643 of Hamamatsu with multi-alcali cathode of $13 \mathrm{~mm}$ diameter. Quantum efficiency of the PMT cathode is $20 \%$ for a wavelength of $350 \mathrm{~nm}$. PMT's multi-alcali cathode (instead of the standard bi-alcali one used in ground-based fluorescence detectors) was chosen for operation in a wider range of temperatures where the cathode operates in a linear regime. To make the detector field of view (FOV) uniformly filled with pixels, light guides with square entrance $(15 \times 15 \mathrm{~mm})$ and circle output adjusted to PMT cathode were used.

The principles of observations using the TUS instrument are illustrated in Figure 1.

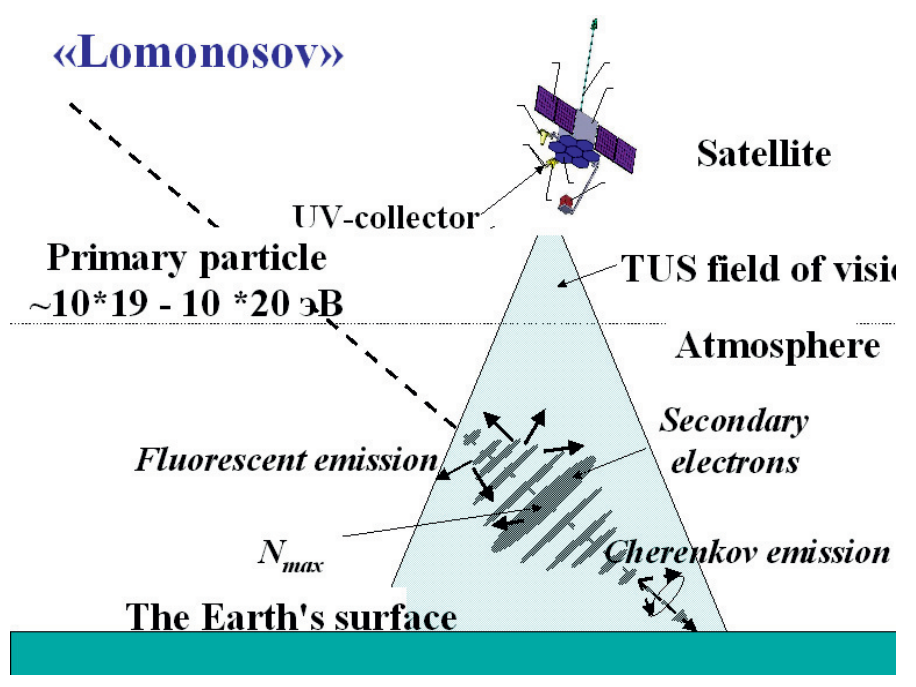

Fig. 1. Illustration of UHECR detection with TUS.

\subsection{Gamma-ray burst monitor BDRG and wide-field optical cameras SHOKs}

The BDRG instrument is intended for monitoring and locating gamma-ray sources at the celestial vault within the gamma-range and for the production of the trigger signal for the SHOCK wide-field optic cameras. 


\section{BDRG provides:}

- monitoring of the transient astrophysical phenomena (GRBs, "X-ray novas", "Soft gamma-ray repeaters", etc.);

- timing of soft gamma-radiation of the X-ray double stars and pulsars;

- patrol of solar radiation within the gamma-range.

The BDRG instrument consists of three identical gamma-ray detector units BDRG-1...BDRG-3 with axes normally directed to each other. The system as a whole allows to observe a half of celestial sphere and to produce a rectangular co-ordinate system with the axes coinciding with the axes of the detectors. Each gamma-ray burst is detected by one of the detectors or by a combination of two or three detectors. In the last case directing cosines which set the location of the source against the detecting system can be determined by the ratio of the counting rate amplitude increases in each detector to the total amplitude (the counting rate) which characterises the total flux falling on the detecting system. This method provides accuracy of the localisation of gamma-ray burst source on the sky for the most powerful events $1^{\circ}-4^{\circ}$ (Mazets \& Golenetskii 1981).

The SHOCK instrument (Russian abbreviation for "Optical camera of superwide field of vision") consists of two stationary wide-angle fast cameras. Their field of view is situated within the area of gamma-bursts' detection of other instruments onboard the "Lomonosov" satellite.

Each SHOK unit is an optical camera with a wide field of view, which must be within the field of view of the corresponding detector of the gamma-bursts monitor. Due to this feature it is possible to detect the burst within the optical and gamma-ray ranges simultaneously, and in the case of continuous observations a significant opportunity for measuring of optical curves of the gamma-ray burst prompt emission and their precursors' detecting are provided.

Field of view of each camera is about 1000 square degrees, and maximum framing rate is about 5-7 frames/sec. In fact, cameras record "a movie" continuously, and in case of gamma-ray burst detection part of this movie can be transmitted to the Earth.

Among the bursts it is possible to process the images in order to find optical transients: supernova, novae, "orphan" bursts, asteroids and near-space objects and space debris.

It must be emphasized that the SHOK device will be the first orbital experiment with cameras of super-wide field. Development of detection methods of dangerous asteroids and space debris from space are of particular interest.

\subsection{UFFO instrument}

The UFFO instrument consists of 20-cm UV-optic telescope SMT with a fast rotating mirror and the wide-field X-ray imager UBAT.

The main goal of the observations by means of the UV-telescope SMT is the recording of the intrinsic radiation of gamma-ray bursts due to opportunity of very 
fast $(\sim 1 \mathrm{~s})$ rotation of the mirror focusing in the region of the burst localisation at the moment of the trigger of gamma-monitor or UBAT instrument which allows to obtain images within X-range (Chen et al. 2011). The UBAT instrument is based on the combination of a coding mask and the position-sensitive detector with pixels produced of LYSO scintillator.

\subsection{Instruments fir magnetosphere study: DEPRON and EIFIN-L}

The DEPRON instrument (Dosimeter of Electrons, PROtons and Neutrons) is intended for measurements of the absorbed doses and linear energy transfer spectra from high-energy electrons, protons and nuclei of space radiation, and for detecting thermal and slow neutrons.

The instrument includes:

- Charged particles dosimeter based on semiconductor detector;

- Thermal neutrons detector based on gas-discharge counter SI13N;

- Circuits for analogous and digital processing of detectors' signals, for information storage and analysis;

- Power supply units for the detectors and electronics.

The ranges of the neutron flux density measured by means of SI13N counters for the neutrons within the energy range of $10^{-3}-10^{2} \mathrm{eV}$ is $0.1-10^{2}$ neutrons $/\left(\mathrm{cm}^{2} \mathrm{~s}\right)$.

The ELFIN-L (Electron Loss and Fields Investigator for the "Lomonosov" mission) instrument is a joint project of the Institute of Geophysics and Planetary Physics at the University of California, Los-Angeles (IGPP/UCLA) and Skobeltsyn Institute of Nuclear Physics of M.V. Lomonosov Moscow State University. It consists of a Flux Gate Magnetometer (FGM), an Energetic Particle Detector for Electrons (EPDE), and an Energetic Proton Detector for Ions (EPDI).

The main scientific objective of the MSU-UCLA collaboration is to understand the dominant mechanisms of the loss of energetic electrons and ions. Energetic particles create a hazardous environment for satellites and humans in space and cause a number of satellite failures.

\subsection{The information unit (BI)}

The Lomonosov on-board equipment also includes the information unit BI (Russian abbreviation for "Information unit"), which is needed in order to provide control of the scientific equipment complex onboard the "Lomonosov" satellite. It provides collection, storage and transmission of the telemetric information to Earth.

An information unit is developed in order to provide operation of the scientific equipment complex onboard the "Lomonosov" satellite and its operative and flexible control during the execution of the scientific program. The service systems of the basic satellite platform can not fit the requirements of the unique scientific 
experiments - complicated scientific equipment, huge volume of scientific information storage, high-operative control of the equipment, therefore it was necessary to develop a special information unit.

\section{Conclusion}

The set of instruments installed on-board the "Lomonosov" satellite allow the study of a wide range of cases of modern astrophysics and space physics. The main of them are the studies of extreme events in sources placed on cosmological distances such as UHECRs and GRBs. To be launched in the nearest future the "Lomonosov" mission gives the scientists a good opportunity for solving the mentioned above problems.

This work was partially supported by funds from Megagranta $\mathrm{N}^{\circ}$ 11.634.31.0076.

\section{References}

Chen, P., Ahmad, S., Ahn, K., et al., 2011 (The UFFO Collaboration), Proc. $32^{\text {nd }}$ ICRC Beijing (August 11-18)

Garipov, G.K., Khrenov, B.A., Panasyuk, M.I., et al., 2005, Astropart. Phys., 24, 400

Garipov, G.K., Khrenov, B.A., Klimov, P.A., et al., 2010, J. Geophys. Res., 115, doi:10.1029/2009JA014765

Gorbovskoy, E.S., Lipunova, G.V., Lipunov, V.M., et al., 2011, MNRAS, in press

Mazets, E.P., \& Golenetskii, S.V., 1981, Astrophys. Space Sci., 75, 47 